\title{
Expandiendo la tradición de Ángel Palerm: estaciones de campo, etnografía, reclutamiento y retención de estudiantes latinos
}

\author{
Laura González y Víctor García
}

\begin{abstract}
El artículo habla de la Casa "Ángel Palerm", un modelo de estación de campo etnográfica que establecimos en Dallas, Texas, para reclutar y retener a estudiantes latinos en la Universidad de Texas en Dallas. Abrió sus puertas en Oak Cliff, uno de los barrios latinos más grandes de Dallas y operó del año 2000 hasta el 2004. Se describe el enfoque, la pedagogía utilizada y su aplicación para llevar a cabo esta misión. La discusión incluye la tradición intelectual detrás de este esfuerzo: la "escuela de antropología" de Ángel Palerm. También se describen las colaboraciones desarrolladas con los colegios comunitarios del condado de Dallas y otras instituciones. La Casa "Ángel Palerm" fue un proyecto financiado por el Departamento de Educación de Estados Unidos, diseñado para preparar, reclutar y retener a estudiantes latinos en las universidades.
\end{abstract}

PALABRAS CLAVE: estaciones de campo, latinos, reclutamiento, Ángel Palerm, colegios comunitarios

\section{Spreading the Ángel Palerm Tradition: Field Schools, Ethnography, and the Recruitment and Retention of Latino Students}

Our article addresses Casa "Ángel Palerm", an ethnographic field school model, established in Dallas, Texas, to recruit Latino students to the University of Texas. It was opened in Oak Cliff, one of the largest Latino barrios in Dallas, from 2000 to 2004. We describe our field school approach, its pedagogy, and its use for this mission. Our discussion also includes the intellectual tradition behind our efforts: the Ángel Palerm "school of anthropology". We describe the field school's collaborations with the Dallas County Community Colleges and other partners of the project. Casa "Ángel Palerm" was a project designed to prepare, recruit, and retain Latino students at the university.

KEYWORDS: ethnographic field schools, Latinos, recruitment, Ángel Palerm, community colleges LAURA GoNZÁLEZ: Indiana University of Pennsylvania, Pensilvania, Estados Unidos
gnzlzlr@gmail.com

Víctor García: Indiana University of Pennsylvania, Pensilvania, Estados Unidos vgarcia@iup.edu

Desacatos, núm. 45, mayo-agosto 2014, pp. 35-46

Recepción: 4 de diciembre de 2012 / Aceptación: 6 de enero de 2013 
$\mathrm{C}$

omo señala Madelyn Iris (2004) al inicio del número Passages: The Ethnographic Field School and First Fieldwork del NAPA Bulletin (National Association for the Practice of Anthropology, NAPA), dedicado a las estaciones de trabajo de campo etnográfico en Estados Unidos, la mayoría de los departamentos de Antropología de ese país no tienen ni utilizan estaciones de trabajo de campo etnográfico en sus programas de estudio. Su uso es más común entre los arqueólogos que entre los antropólogos sociales o culturales. La autora afirma que los departamentos que sí incluyen el trabajo de campo etnográfico como parte de la formación de sus estudiantes lo hacen porque consideran que ésa es la mejor forma de enseñarles los métodos de investigación y de exponerlos a otras culturas. Nosotros también sabemos por experiencia propia que las estaciones de trabajo de campo etnográfico son de gran relevancia en la formación de estudiantes de licenciatura y posgrado. ${ }^{1}$ Pero lo más importante en el caso que aquí presentamos es su

${ }^{1}$ Laura González fue alumna de Ángel Palerm de 1971 a 1979. Realizó prácticas y estancias de campo en las estaciones de campo que la Universidad Iberoamericana instaló en Tepetlaoxtoc, Estado de México; Lagos de Moreno, Jalisco; Ciudad Victoria, Tamaulipas, y Valle de Santiago, Guanajuato. Más tarde, primero como becaria del Centro de Investigaciones Superiores del Instituto Nacional de Antropología e Historia (CIS-INAH) y luego como profesora-investigadora de la Universidad Autónoma Metropolitana-Iztapalapa (UAM-I), dirigió la estación de campo ubicada en San Lorenzo, Huehuetitlán, Estado de México. En 1984 se trasladó a la Universidad de California, Santa Barbara, con residencia en Guadalupe y Santa María, California, donde continuó con sus estudios de posgrado mientras colaboraba en la formación de equipos de estudiantes que hacían recorridos y prácticas de campo en los valles centrales y costeros de California. En 1994, como profesora-investigadora de la Universidad de Guanajuato, estableció y dirigió las estaciones de campo de Moroleón y Yuriria, Guanajuato. En 1998 fue invitada por la Universidad de Texas en Dallas para continuar con la investigación sobre redes internacionales de migrantes que llevaba a cabo desde Guanajuato. En 2000 estableció la Casa "Ángel Palerm", estación de campo que empezó a operar en el barrio Oak Cliff desde finales de 1998 y que después se convirtió en el Centro Oak Cliff para el Estudio de las Comunidades (The Oak Cliff Center for Community Studies, occcs), una organización sin fines de lucro dedicada a empoderar a los latinos a través de su acceso a la educación superior y el involucramiento con sus comunidades - "empowering Latinos through higher education and community involvement"- (Cátedra "Ángel Palerm”, 2012). utilidad para reclutar y retener a estudiantes latinos en universidades y colegios comunitarios, así como para vincular social y culturalmente a los estudiantes y sus familias con las instituciones de enseñanza superior.

A lo largo de los últimos 30 años hemos enseñado a hacer trabajo de campo etnográfico en las estaciones de campo que hemos instalado, tanto en Estados Unidos como en México, a fin de capacitar a estudiantes, principalmente antropólogos, en el método etnográfico. Sin embargo, en el año 2000 iniciamos el uso de este modelo de estación de campo permanente en Dallas, Texas, con otro propósito. Nuestro artículo trata de este enfoque y del proyecto de transferencia Fund for the Improvement of Postsecondary Education (FIPSE), novedoso entonces y ahora porque uno de los objetivos, además de formar investigadores en ciencias sociales con los métodos tradicionales de la etnografía, fue reclutar y retener a estudiantes latinos en las universidades y colegios comunitarios de la región de estudio. Lo que hicimos fue incorporar a los estudiantes latinos, a "los sujetos de estudio", como ayudantes de investigación de nuestros propios proyectos, en particular en la investigación sobre las barreras y obstáculos que ellos mismos enfrentaban para ingresar y luego graduarse en las universidades. A continuación describimos este enfoque de trabajo de campo, la pedagogía utilizada y su aplicación para llevar a cabo esta desafiante misión. La discusión incluye la tradición intelectual detrás de este esfuerzo, la “escuela de antropología” de Ángel Palerm. Esta escuela de pensamiento y "especial manera de hacer antropología” enfatiza la investigación in situ, basada en el uso de una estación de campo permanente instalada en la comunidad de estudio, en la cual se capacitan estudiantes al mismo tiempo que se realiza investigación sobre asuntos clave y se debaten los problemas de la comunidad y de la región.

\section{ANTROPOLOGÍA, DIVERSIDAD Y LOS LATINOS}

Durante varias décadas las universidades de Estados Unidos tanto privadas como públicas han tratado 
de diversificar su población estudiantil. No es común el uso de la antropología para reclutar a minorías estudiantiles y miembros de otras poblaciones en desventaja a pesar de que la antropología es la disciplina idónea para llevar a cabo esta tarea. La antropología social o cultural se enorgullece de no sólo ocuparse de estudiar cuestiones de origen étnico, racial, de género y clase, sino de incluir contribuciones de los grupos que carecen de representación en la disciplina, como nosotros, los antropólogos latinos en Estados Unidos. La disciplina antropológica también alienta a los estudiantes a pensar críticamente sobre su responsabilidad social y su participación cívica, y los prepara para resolver problemas sociales que enfrentan muchas poblaciones en situaciones de desventaja. ${ }^{2}$

En un ensayo titulado "Bringing Anthropology Home: Latino/a Students, Ethnographic Research, and us Rural Communities", uno de nosotros (García, 2001) reflexionó en torno al estancamiento $\mathrm{y}$ al reducido número de latinos doctorados en antropología prevaleciente en Estados Unidos, así como respecto de algunas de las razones que originan este hecho lamentable. El documento fue presentado en 1997 en la Conferencia Transforming the Social Sciences through Latino Studies, patrocinada por el Julian Samora Research Institute (JSRI), de la Universidad Estatal de Michigan, y fue publicado cuatro años más tarde. Lo más importante de ese artículo fue el planteamiento de una posible solución al problema del reducido número de antropólogos latinos en Estados Unidos. Se propuso una pedagogía que convirtiera al estudiante en participante activo de su propia formación mediante un programa de capacitación etnográfica similar al desarrollado en México en la década de

\footnotetext{
2 Víctor García lleva más de 20 años enseñando, escribiendo y enfatizando la importancia de la investigación participativa, llamada en la actualidad "antropología activista" - "action research" o "engaged anthropology" - (García, 2001, 2002, 2003; García y González, 2009, 2011; página electrónica MARTI).
}

1970 por el antropólogo Ángel Palerm, ${ }^{3}$ vigente en la Universidad Iberoamericana (UIA), Campus Santa Fe. Este programa, que se discute ampliamente en los otros artículos de este número de Desacatos, incluye la realización de seis semanas de trabajo de campo al inicio del programa de estudios para los estudiantes del posgrado en antropología y el uso de una estación de campo permanente en la que se enseñan los métodos etnográficos.

\section{EL MODELO DE ESTACIÓN DE CAMPO}

Ángel Palerm, fundador de esta escuela mexicana de antropología, fue un defensor de la integración de la teoría y la práctica al inicio de la carrera del estudiante. Para este propósito estableció una estación de campo en Tepetlaoxtoc, un pueblo cerca de

\footnotetext{
${ }^{3}$ Los logros de Ángel Palerm fueron resumidos por su amigo y colega Eric Wolf (1981) en un obituario publicado en American Anthropologist. Jorge Alonso escribió en 1999 una pieza corta sobre las contribuciones de Palerm a la antropología mexicana en el primer volumen de la Serie Biografías del Colegio de Etnólogos y Antropólogos Sociales. Un recuento detallado de sus aportaciones se encuentra en un libro homenaje a cargo de Susana Glantz (1987a), La heterodoxia recuperada: en torno a Ángel Palerm. Los aportes de Palerm al pensamiento de la antropología contemporánea pueden consultarse en los dos volúmenes de ensayos Historia, antropología y política: homenaje a Ángel Palerm, compilados por Modesto Suárez (1990). Virginia García Acosta (2000) coordinó y escribió la introducción del volumen $L a$ diversidad intelectual. Ángel Palerm in memoriam. A la autoría de Patricia Torres (1988) se debe el texto "Ángel Palerm Vich". También recomendamos la lectura de "Ángel Palerm y la institucionalización de la antropología social en México", de Luis Vázquez León (1996), y de "La familia dividida y los círculos invisibles: las instituciones y los estudios de posgrado en antropología en México", de Teresa Rojas Rabiela (1997). Carmen Viqueira escribió "Algunas experiencias sobre la enseñanza de la antropología social en México" y Juan Vicente Palerm "Las nuevas comunidades mexicanas en espacios rurales de los Estados Unidos de América: a propósito de una reflexión acerca del quehacer antropológico", ambos textos publicados en 1999 en Áreas. Revista de Ciencias Sociales. Estas referencias pueden consultarse en la página <www.ciesas.edu.mx/catedra_palerm/index.html> y en la Biblioteca "Ángel Palerm", ubicada en Tlalpan, en la ciudad de México, lo mismo que el disco compacto de la Cátedra "Ángel Palerm" 2012.
} 
Texcoco - actualmente la Casa "José de Acosta", conocida por todos como la Casa de Tepetla-, donde hasta hoy muchos estudiantes mexicanos y extranjeros aprenden el arte de la investigación etnográfica al tiempo que examinan problemas reales y trabajan sus temas de interés dentro de los proyectos de investigación que dirigen sus profesores.

Como lo describen Marisol Pérez Lizaur y Roger Magazine en las primeras contribuciones que integran la sección "Saberes y razones" de este número de Desacatos, desde la apertura de la estación de campo o Casa de Tepetla entre 1966 y 1967 se han formado muchas generaciones de estudiantes de la UIA y de otras universidades de América Latina, Estados Unidos, Europa y Asia. La formación que reciben actualmente los estudiantes inscritos en los programas de maestría y doctorado del Departamento de Ciencias Sociales y Políticas de la UIA no puede ser la misma que la que obtuvimos en los años setenta del siglo anterior al hacer trabajos de campo desde la Casa de Tepetla. Ya no existe la licenciatura en antropología y el Consejo Nacional de Ciencia y Tecnología (Conacyt) exige que los estudiantes terminen sus programas de estudios en dos años.

En las décadas de 1970 y 1980, como lo detalla Jacinta Palerm (1997), los estudiantes formados en ese modelo pasábamos semanas o meses en el campo. Todos los días estábamos ocupados con talleres, o en recorridos de campo y en sesiones en las que recibíamos instrucciones y presentábamos informes o que dedicábamos a animados debates. Amigos y colegas de Ángel Palerm, reconocidos antropólogos estadounidenses, como Eric Wolf, Richard Adams, Sidney Mintz y William Sanders, nos visitaban y contribuían a las discusiones del día. Profesores y estudiantes formábamos equipos y reflexionábamos sobre temas específicos, como la tenencia de la tierra, los sistemas agrícolas, los sistemas de parentesco y la migración laboral. Los estudiantes, ya sea solos o con los profesores, visitábamos diariamente o vivíamos en las comunidades rurales cercanas. Estas excursiones nos introdujeron a los rigores del trabajo de campo y nos permitieron aplicar lo que habíamos aprendido en los talleres. En las tardes, estudiantes y profesores, como grupo, discutíamos los hallazgos del día, analizábamos la información adquirida así como los problemas éticos que enfrentábamos al interactuar con nuestros informantes y hasta con los propios compañeros y maestros.

El modelo de estación de campo utilizado por el proyecto de transferencia FIPSE fue adoptado a partir de otro proyecto del mismo fondo, el "Mexican Immigration and Chicano/Mexican Population Growth in Rural California Project", desarrollado por Juan Vicente Palerm, hijo de Ángel, a principios de la década de 1980. Juan Vicente Palerm era y es profesor del Departamento de Antropología de la Universidad de California, en Santa Barbara (UCSB), y en ese momento era director del Centro de Estudios Chicanos de la misma universidad. El objetivo del proyecto era retener a los estudiantes de licenciatura y de maestría de los departamentos de Historia, Ciencias Políticas, Sociología y Antropología con base en tutorías de investigación, a través de las cuales éstos y sus profesores se organizaban en equipos de investigación de los tres niveles y trabajaban juntos en un proyecto de investigación mayor.

Se ofrecía un seminario a la semana diseñado para ayudar a los estudiantes a desarrollar sus proyectos de investigación, discutir la literatura, planear recorridos de campo y aprender sobre la ética de la investigación y los métodos del trabajo de campo. Los estudiantes tenían la oportunidad de llevar a cabo su entrenamiento en el campo. Los recorridos a las comunidades de trabajadores agrícolas cerca de la UCSB se realizaban cada dos semanas a lo largo del trimestre académico regular y más a menudo durante el verano. También se estableció una estación de campo en una comunidad rural cercana, donde vivíamos los coautores de este artículo -entonces un par de estudiantes del posgrado en antropología- y a donde podían llegar a visitarnos los otros estudiantes que deseaban estudiar el área. Nuestro departamento se utilizaba asimismo para reuniones, para almacenar datos de campo y para legitimar la presencia de los estudiantes e investigadores 
de la UCSB en la comunidad. A partir de esta estación de campo instalada en Guadalupe, California, se hicieron otros recorridos de campo más largos para explorar sistemas agrícolas en diferentes regiones ecológicas del valle de San Joaquín. Exponer a los estudiantes de licenciatura y posgrado al trabajo de campo al inicio de sus estudios era algo nuevo para el programa de antropología cultural en la UCSB. En general, en Estados Unidos los estudiantes, en especial los de licenciatura, no realizan trabajo de campo sino hasta que están en cursos de posgrado y sólo después de haber avanzado hacia la candidatura al doctorado, es decir, luego de haber aprobado todos los cursos y un examen muy difícil.

\section{ETNOGRAFÍA, CASA “ÁNGEL PALERM” Y LA COMUNIDAD LATINA EN DALLAS}

En 1999, transcurridos dos años desde la conferencia del JSRI, instrumentamos algunas de las recomendaciones propuestas en el documento mencionado en un proyecto de investigación y formación de estudiantes de la Facultad de Ciencias Sociales de la Universidad de Texas en Dallas (UTD). Una recomendación importante fue utilizar la etnografía y una estación de campo permanente ubicada en la comunidad bajo estudio para hacer que la antropología y la educación universitaria fueran significativas y relevantes para los estudiantes latinos. A un equipo de profesores e investigadores de dicha Facultad se le concedió una subvención de cuatro años por parte del FIPSE, programa del Departamento de Educación de Estados Unidos que tenía por objetivo transferir estudiantes latinos de los colegios comunitarios del distrito y condado de Dallas (Dallas County Community College District, DCCCD) a la UTD y a otras universidades de la región. ${ }^{4} \mathrm{El}$

\footnotetext{
${ }^{4}$ Los profesores que participamos en el Proyecto FIPSE fuimos el doctor Bobby Alexander, profesor de sociología de la Escuela de Ciencias Sociales de la UTD, el doctor Geoffrey Grimes, profesor de inglés en Mountain View College, y nosotros, la doctora Laura
}

Proyecto FIPSE "Un modelo etnográfico de ciencias sociales basado en la comunidad con el fin de reclutar y retener latinos" se dedicó a superar cuatro obstáculos principales para la transferencia: las barreras monetarias, sociales y culturales; la falta de claridad; la falta de relevancia de la educación superior, y la preparación insuficiente de los estudiantes para tomar cursos de nivel universitario (Alexander et al., 2003 y 2007).

El núcleo del Proyecto FIPSE fue un curso de estudios culturales que ofrecimos en Mountain View College (MVC), "Cultural Studies 2370: Fiesta Oak Cliff y la estación de campo etnográfico" (García, González y Grimes, 2004a). El Mvc, situado en el barrio Oak Cliff, fue y sigue siendo uno de los colegios comunitarios con la mayor proporción de estudiantes latinos en el DCCCD. ${ }^{5}$ Oak Cliff es el barrio con la concentración más grande de latinos en Dallas y se encuentra al centro del área de servicio del colegio. En general, son las bajas tasas de transferencia de estudiantes latinos que exhiben el MVC y el conjunto de DCCCD las que impiden que los estudiantes latinos entren y se gradúen de las universidades del área.

El programa del curso "Estudios Culturales 2370: Fiesta Oak Cliff" se concentró en enseñar etnografía y demostrar su valor en la enseñanza y aprendizaje de la cultura local de los latinos, así como en la motivación de los estudiantes de este grupo poblacional para continuar su educación más allá del colegio comunitario. Una parte del curso consistía en sesiones interactivas con la participación de oradores invitados, en animadas discusiones sobre las lecciones y las tareas, en talleres sobre el método

González, antropóloga, profesora-investigadora en la Escuela de Ciencias Sociales de UTD, y el doctor Víctor García, profesor de antropología y actualmente también director del MARTI de la Universidad de Indiana en Pensilvania (IUP).

${ }^{5}$ MvC es una "institución de servicio hispana" - "Hispanic serving institution", HSI- y como tal es elegible para recibir subvenciones y asistencia federal adicional. Para que un colegio comunitario o universidad sean reconocidos como HSI, los estudiantes hispanos deben representar, al menos, $25 \%$ del total de la inscripción estudiantil. 
etnográfico en la estación de campo Casa "Ángel Palerm" y en las excursiones o recorridos de campo por el barrio Oak Cliff. El curso atrajo a un diverso grupo de estudiantes, de entre los cuales la mayoría de los matriculados eran latinos. Estos últimos provenían de Oak Cliff, aunque, al igual que muchos otros jóvenes, no eran miembros activos de sus comunidades y no siempre se involucraban en las actividades y eventos locales. Para familiarizarlos con sus comunidades y para que tomaran conciencia de la difícil situación educativa que vive la población local el curso incluyó recorridos de campo y visitas a la estación de campo Casa "Ángel Palerm", ubicada en el centro de la comunidad, a la cual asistían también líderes comunitarios y otros estudiantes y colegas que vivían en la estación de campo o que nos visitaban durante algunas semanas o meses.

Como explicaremos más adelante, la Casa "Ángel Palerm" o estación de trabajo de campo etnográfico permanente que instalamos en el 2000 fue el componente más importante de nuestros proyectos de investigación y de los cursos que impartimos tanto en el MVC como en la UTD. Nos asentamos en una vivienda unifamiliar típica del barrio Oak Cliff el 15 de abril de 2000. Durante exactamente dos años hicimos trabajo de campo y vivimos en otra casa, también en Oak Cliff, más pequeña e incómoda, desde donde realizábamos investigaciones sobre el tamaño y la composición de la red Gua-Tex de migrantes internacionales, que une Guanajuato con Texas, que contrastábamos con las redes Gua-Pa y Cali-Gua, que unen a Guanajuato con Pensilvania y con California, respectivamente. Desde 1994 empezamos a estudiar estas redes con apoyo de la Universidad de Guanajuato y la Oficina de Atención a Guanajuatenses en el Exterior del Gobierno del Estado de Guanajuato. Los coautores de este artículo vivimos en ambas estaciones de campo con otros profesores-investigadores de la UTD, varios estudiantes de posgrado de universidades mexicanas y estadounidenses, además de colegas antropólogos, politólogos y sociólogos de otros países que nos visitaban con frecuencia.
El 10 de junio de 2000, exactamente 20 años después del fallecimiento de nuestro querido maestro Ángel Palerm, inauguramos la nueva estación de campo con una ceremonia muy especial, en la que algunas autoridades locales pronunciaron discursos y para la cual se montó una impresionante exhibición de fotografías del artista guanajuatense Javier Zamora y se contó con presentaciones de profesores y alumnos de la uiA, de la Universidad de Guanajuato y de la UTD. Ese día disfrutamos de una rica cena con tamales y develamos una placa de azulejos, elaborada en Dolores Hidalgo, Guanajuato, que decía: "Estación de Campo Ángel Palerm de Dallas, Tx, junio 2000" (“Casa Ángel Palerm de Dallas, Texas", Cátedra “Ángel Palerm”, 2012).

Los latinos de Oak Cliff, la mayoría inmigrantes mexicanos y sus hijos, componían $44.5 \%$ de los 326 840 residentes de la comunidad. Un número significativo - 35\% - de los hogares en Oak Cliff norte vivían en niveles considerados de pobreza (United States Census Bureau, 2003b). Además, la población era joven: 66356 residentes - 55\% — tenían 24 años o menos (United States Census Bureau, 2003a). De los 94809 residentes mayores de 25 años, 53\% no había completado la escuela preparatoria o high school, 22\% la había terminado, pero no asistía a colegios comunitarios ni universidades, y el resto asistía a la universidad o había obtenido un título universitario (United States Census Bureau, 2003c).

La Casa "Ángel Palerm" de Dallas, Texas, fue utilizada para realizar distintas actividades relacionadas con el Proyecto FIPSE. Fue aula de y en la comunidad. Sirvió de base para los recorridos de campo. En ella podían encontrarse los estudiantes participantes que hacían investigaciones en la comunidad, tanto como fuese posible, como estudiantes-investigadores (García, González y Grimes, 2004b). Aparte de funcionar como un lugar para la investigación y la instrucción, era un espacio de encuentro, donde estudiantes y profesores se reunían para discutir sus proyectos de familia, para debatirlos, así como para llevar a cabo actividades de otras asignaturas. Los estudiantes de los niveles de licenciatura 
y posgrado de distintas disciplinas académicas de la UTD también hicieron uso de la estación de campo. Estos estudiantes desarrollaban sus protocolos de investigación, reclutaban informantes y ahí mismo realizaban las entrevistas para sus proyectos. Los materiales que guardamos en la estación de campo, como artículos, libros, fotografías, mapas, videos y cientos de genealogías, estaban a disposición de los estudiantes. En la Casa "Ángel Palerm" también se impartieron conferencias a cargo de investigadores invitados y líderes de la comunidad, de otras ciudades de Texas, California, Pensilvania, Chicago y de otros países, como Australia, Suecia y México. Los estudiantes presentaban sus trabajos con la asistencia de sus compañeros, profesores, familiares, vecinos y otros miembros de la comunidad. La estación de campo fungía a su vez como centro comunitario y en ella los estudiantes y sus padres se enteraban de oportunidades de educación, como becas y ferias educativas, y se percataban de la importancia de continuar su educación superior más allá del colegio comunitario. Todos nos visitaban, telefoneaban y se comunicaban con mucha frecuencia por medio de correo electrónico y visitas que hacían con sus familiares, compañeros, vecinos y amigos.

La parte clave del curso de estudios culturales fue el Proyecto Familias, a través del cual los alumnos estudiaban dos familias, la propia y otra. Debido a su diseño, el proyecto incluía a las familias de los estudiantes y a otros miembros de su comunidad. Con los métodos de campo aprendidos en los talleres, como la observación participante, la entrevista y la elaboración y análisis de las genealogías, los estudiantes investigaban el origen y evolución de su propia familia, el nivel educativo de todos sus integrantes y sus ocupaciones, así como la historia de la migración. Además examinaban a otra familia latina no relacionada con ellos. Sus investigaciones les mostraron una correlación entre los bajos niveles de educación en sus familias - muy pocos miembros tenían un título universitario o un diploma de escuela secundaria- y las limitadas oportunidades de empleo. Mediante esta investigación los estudiantes aprendieron los beneficios a largo plazo de continuar con su educación superior. Muchos de los padres migrantes, por ejemplo, si habían sido afortunados, completaron el sexto grado de primaria. Al realizar estas investigaciones independientes, como la del Proyecto Familias, tuvimos la oportunidad de enseñar a los estudiantes cómo hacer investigación antropológica, cómo usar el método comparativo, lo mismo que desarrollar sus habilidades de lectura, escritura y presentación oral. Incluimos los resultados de las investigaciones de los estudiantes en nuestro propio proyecto de investigación sobre los latinos y las barreras para su educación superior.

El Proyecto Familias, más que ninguna otra de las secciones del curso, ayudó a los estudiantes latinos a superar dos de los cuatro obstáculos del proyecto: los relacionados con la importancia de la educación superior y la insuficiente preparación para tomar cursos de nivel universitario. Gracias a las investigaciones que hicieron sobre sus familias, los estudiantes obtuvieron una visión más clara sobre la relevancia de una educación universitaria y aprendieron a desarrollar habilidades analíticas y de escritura. Trabajamos en estrecha colaboración con los estudiantes en la preparación de sus trabajos escritos y en sus informes finales. Como los profesores somos bilingües y biculturales pudimos atender a los estudiantes y a sus familiares tanto en inglés como en español. Los estudiantes recibieron asesoría y se les alentó a buscar clases compensatorias de escritura y lectura en inglés en el MVC. Este mismo proyecto, con otras actividades del curso, como visitas a la UTD y reuniones con profesores y personal administrativo de las universidades, permitió superar el cuarto obstáculo que habíamos identificado: las barreras monetarias, por medio de becas, incluyendo las exenciones de colegiaturas para los estudiantes latinos que se transfirieron exitosamente del MVC a la UTD. Algunos estudiantes también fueron contratados como ayudantes de investigación de la Casa "Ángel Palerm" gracias a los recursos del Departamento de Educación transferidos a la UTD a través de nuestro Proyecto FIPSE. 


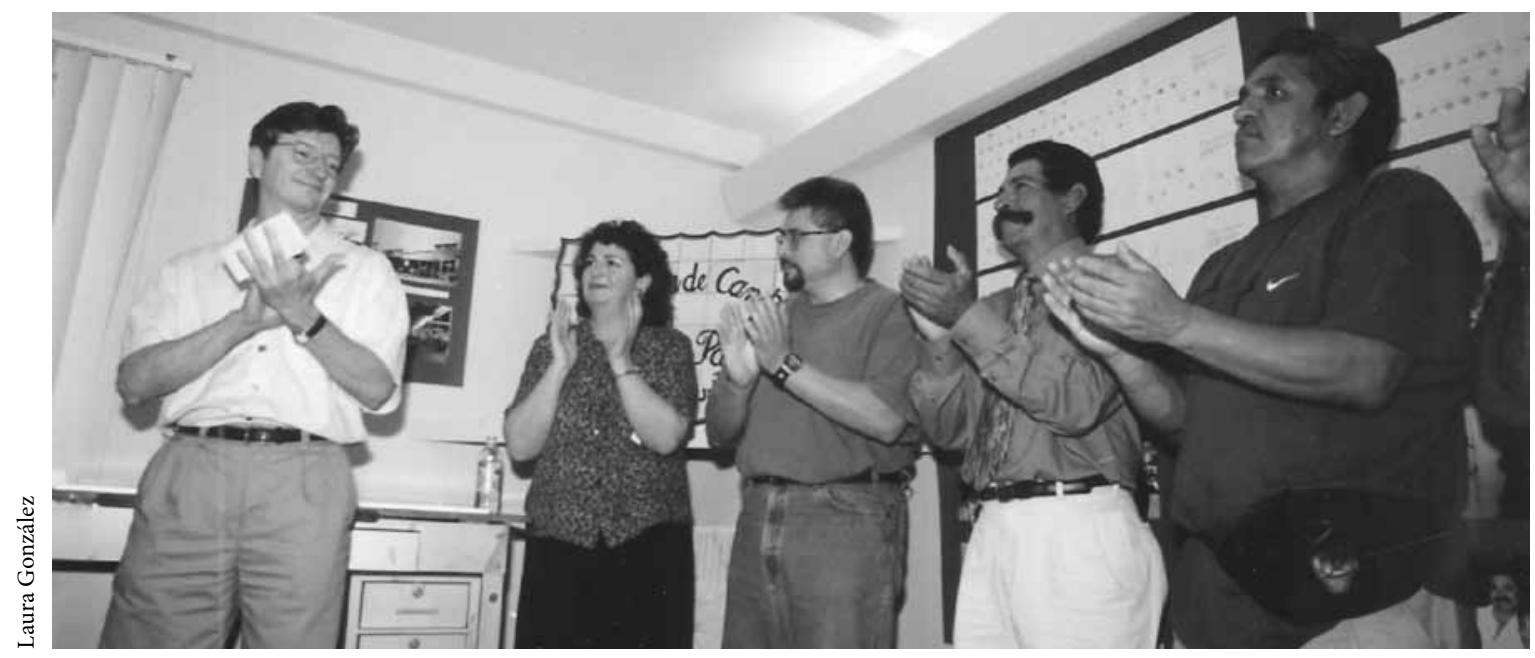

Inauguración de la Casa “Ángel Palerm”, 10 de junio de 2000.

\section{ÉXITOS DE LA ESTACIÓN DE CAMPO Y LA EDUCACIÓN DE LOS LATINOS}

El arraigo que adquirió la Casa "Ángel Palerm” en la comunidad puso al descubierto otros obstáculos que eran insuperables para los estudiantes latinos, además de los cuatro estudiados por el Proyecto FIPSE: la situación legal o el estatus de indocumentados de algunos, la necesidad de trabajar para ayudar económicamente a sus familias - algunas veces, con más de un empleo-, roles culturales específicos de género basados en responsabilidades familiares que impedían a las alumnas continuar con su formación profesional y la mala educación que muchos recibieron en las escuelas de donde provenían, lo cual resultó ser el obstáculo más difícil de todos. Éstos y otros aspectos impiden a algunos estudiantes latinos completar su educación en el MVC y proseguir con sus estudios universitarios. Los hallazgos fueron presentados primero en una investigación comisionada por el Proyecto de Derechos Civiles de la Universidad de Harvard y el Pew Hispanic Center, y luego fueron publicados en el Journal of Hispanic Higher Education, en el Community College Enterprise, TACHE Noticiario y en Teaching Anthropology: Publication of the Society for Anthropology in Community Colleges. Con ello se pretendía una mejor comprensión de las abrumadoras dificultades que deben superar los estudiantes latinos, en especial los inmigrantes, para continuar con su educación superior.

En cuanto a los resultados, de los 131 estudiantes que se inscribieron en el curso que se ofreció por primera vez en la primavera de 2001 y hasta la primavera de 2004, 31 obtuvieron un diploma o certificado en el MVC. Al finalizar la primera fase del Proyecto FIPSE, el colegio siguió ofreciendo el curso como parte de sus clases regulares. Durante el año académico 2004-2005 se inscribieron 61 estudiantes. El Proyecto FIPSE tuvo éxito al retener a los estudiantes que tomaron el curso: $79 \%$ en 2003 y $60 \%$ en 2004. Las tasas de graduación también fueron altas. Las tasas de transferencia no pueden medirse con precisión porque la Junta de Coordinación de Educación Superior del Estado de Texas y el DCCCD no hacen públicos estos datos. Varias docenas de estudiantes con quienes mantuvimos contacto nos informaron que se habían inscrito en universidades de la región, algunos ya habían terminado sus licenciaturas, otros pocos obtuvieron una maestría y sabemos que tres de ellos están terminando programas de doctorado.

La estación de campo etnográfico que se estableció en un barrio residencial de la ciudad de Dallas contribuyó a nuestro éxito, porque facilitó el 
contacto con los estudiantes, con sus familias y con otros miembros de la comunidad, lo que favoreció el desarrollo de una relación estrecha, de confianza y apoyo. Todos ellos se sentían muy cómodos al acudir a la estación de campo en busca de asistencia, ya que se localizaba en su propia comunidad y, sobre todo, porque sentían confianza con nosotros, personas que hablaban su misma lengua y que conocían sus lugares de origen. Con el tiempo, la estación de campo se convirtió en un "hogar lejos del hogar" donde los estudiantes y sus familias podían buscar y conseguir más asesoramiento. Además de enseñar y servir como mentores de los estudiantes durante las horas de clase, también lo hacíamos después, en las tardes y durante los fines de semana. Asesoramos a los estudiantes de manera individual, auxiliamos a sus padres a llenar y entender las solicitudes de ingreso a las universidades o para obtener becas e igualmente les ayudamos a resolver otras necesidades individuales, como convencer al papá para que dejara estudiar a su hija, al esposo para que dejara estudiar a la esposa, al novio para que dejara estudiar a la novia.

La estación de campo operó por otra parte como puente entre las instituciones de educación superior asociadas a través del Proyecto FIPSE: la UTD, el MVC, los latinos y las comunidades de inmigrantes latinos. La Universidad de Texas en Oak Cliff —uT Oak Cliff, como algunos vecinos la llaman - se constituyó como un espacio "neutral" ubicado en el corazón de Dallas en el que se podían discutir asuntos importantes para la comunidad, un lugar en el que latinos y latinas, migrantes, chicanos, estudiantes, sus padres, los líderes comunitarios, profesores de diversas universidades, investigadores de distintas disciplinas y países, miembros de iglesias y partidos políticos, y vecinos con ganas de ayudar se reunían a menudo para intercambiar información y para escuchar las presentaciones de nuestros estudiantes o para atraer a otros posibles estudiantes.

Las actividades de la estación de campo y otras que se llevaron a cabo ahí en conjunción con el curso de estudios culturales hicieron posible la creación de una red social muy fuerte entre los estudiantes y sus familias, los integrantes del Proyecto
FIPSE, otros colegas, autoridades, administradores y líderes de organizaciones comunitarias locales. $\mathrm{Al}$ conectar a todos estos individuos, instituciones y organizaciones involucrados en la educación universitaria de los estudiantes creamos una red social que permitió una distribución más efectiva y eficiente de la información sobre los recursos que apoyan la transferencia de estudiantes a las universidades y que promueven su éxito en las mismas. La estación de campo se consolidó como un recurso primario y fundamental de información y asistencia, y colocó a la coautora de este texto como un contacto vital de la red social de la comunidad latina en Oak Cliff y de la zona metropolitana de Dallas-Fort Worth-Denton.

El mayor testimonio del éxito de la Casa "Ángel Palerm" fue que los estudiantes, sus padres y diversos miembros de la comunidad la reclamaron como suya. Algunos la bautizaron como "la casa de los antropólogos", otros la llamaron "UT Oak Cliff” y quienes estuvieron presentes durante su inauguración le decían "la Casa Ángel Palerm". El sentido de propiedad de la comunidad llevó a instar al equipo del Proyecto FIPSE a continuar sus actividades una vez concluido el proyecto de investigación en agosto de 2004. A instancias de la comunidad, en octubre de 2003, el equipo estableció el Oak Cliff Center for Community Studies (occCs), y un año después obtuvo la exención de impuestos, propia de una organización sin fines de lucro, categoría 501 (c) (3). Laura González se convirtió en la directora ejecutiva del occcs y los otros miembros del equipo FIPSE fungieron como directores del Consejo Directivo. El occcs, que operaba desde la Casa "Ángel Palerm", capitalizó su éxito y amplió el alcance del proyecto para desarrollar programas adicionales, como "Latinas hacia la universidad", "College Prep 101" y "Los primeros pasos o el ABC para ir a la universidad”. Mediante estos programas, González, sus estudiantes y asistentes siguieron asesorando a los padres de familia sobre cómo ser solidarios con la educación de sus hijos. Les ayudaron a afrontar y superar cuestiones personales y familiares muy difíciles que interferían con la educación de sus hijos. 
El occcs fue capaz de proporcionar servicios educativos inestimables, como becas, madrinazgos y subvenciones a la comunidad, hasta febrero de 2009, cuando el centro cerró sus puertas debido a la falta de financiamiento por causa de la recesión de la economía nacional.

El inmueble en el que operó la Casa "Ángel Palerm" y luego el occcs fue primero rentado por Laura González con recursos del Proyecto FIPSE. En julio de 2004 lo compró Jane Bayes, miembro del Consejo Directivo, quien visitaba frecuentemente el Centro y ha apoyado de muy distintas formas todos los programas y proyectos desarrollados por nuestros equipos de investigación desde 1998 y hasta la actualidad. Al momento de cerrar definitivamente el occcs, los programas de atención a los estudiantes latinos y sus familias se institucionalizaron, con lo que adquirieron un carácter permanente y se expandieron a otros colegios, universidades y centros comunitarios del norte de Texas y en Pensilvania. Algunos de estos proyectos de investigación y de apoyo a los estudiantes latinos para seguir sus estudios superiores continúan desarrollándose ahora a partir del Mid-Atlantic Research and Training Institute (MARTI), de la Universidad de Indiana, Pensilvania, y en su estación de campo instalada en la comunidad de Kennett Square en ese mismo estado de la Unión Americana.

\section{CONCLUSIÓN}

Las estaciones de campo muchas veces se usan para enseñar a los estudiantes de antropología los métodos cualitativos de la antropología, para recabar información en escenarios reales y para proveerles de una experiencia intercultural. Como resultó con el proyecto UTD-FIPSE-MvC, la Casa "Ángel Palerm" también fue muy útil no sólo para continuar con la investigación sobre las redes y enclaves de migrantes internacionales que inició González cuatro años antes en la Universidad de Guanajuato, sino también -lo más importante y novedoso- para reclutar y retener a estudiantes provenientes de poblaciones poco representadas en las universidades. La Casa "Ángel Palerm" de Dallas, Texas, y el curso de estudios culturales en el MvC incorporaron a los estudiantes latinos, a sus familias y a los educadores como colaboradores del mismo proyecto de investigación con una meta común. Nuestro modelo de estación de campo proveyó apoyo emocional, psicológico y comunitario a los estudiantes, y al mismo tiempo les ofreció instrucción en el uso de conceptos y terminología científica, lectura crítica y habilidades de escritura técnica para efectuar investigaciones mediante el Proyecto Familias, un ejercicio que impactó directamente sus vidas. Este modelo conectó a las instituciones de educación superior con las comunidades latinas y puso de manifiesto las percepciones que cada uno tenía del otro. Lo principal en todo caso fue que demostró que sí es posible que los latinos obtengan una educación universitaria. Les proveyó un puente para la transición entre instituciones de educación superior de dos años — colegios comunitarios- a instituciones de cuatro años - universidades- para obtener grados de bachelors of arts $\mathrm{y}$ bachelors of sciences, casi equivalentes a las licenciaturas en filosofía, letras, ciencias sociales, ciencias exactas y otras áreas relacionadas.

La Casa "Ángel Palerm” y sus muchas actividades educativas inculcaron en la comunidad latina de Oak Cliff un conocimiento cultural que faltaba en la mayoría de las familias de inmigrantes y en otras familias de bajos recursos, un capital esencial para estimular la transición de sus comunidades hacia la educación superior. Al mismo tiempo, con instrucción académica intensiva y asesoría, los estudiantes latinos continuaron su educación más allá del colegio comunitario. Los modelos UTD y UCSB de los proyectos financiados por FIPSE, basados en la "escuela de antropología” de Ángel Palerm, fueron empleados sobre todo por antropólogos para asistir a los estudiantes latinos. Este mismo modelo puede aplicarse en la enseñanza de otras disciplinas académicas para alcanzar con éxito a otras poblaciones poco representadas y para incluirlas no sólo como sujetos de estudio sino como estudiantes e investigadores de y en sus propias comunidades. 
Esta experiencia, tal como la vivimos de primera mano, llena de fuerza o empodera a los integrantes de estos grupos poblacionales porque incrementa su conocimiento sobre el proceso de educación superior, lo mismo que el de las personas que trabajan en las instituciones de educación superior y están interesadas en reclutar y retener a los jóvenes latinos.

El título original de nuestro artículo iniciaba, a propósito, con la palabra "continuando" y no "expandiendo", "ampliando" o "mejorando" la tradición de Ángel Palerm. ¿Por qué? Porque en la Casa de Tepetla que estableció Palerm aprendimos este modelo que muchos años después replicamos en Guanajuato, en Texas, en California y que seguimos usando en Pensilvania. En todos estos lugares hicimos lo mismo: integrar la docencia y la investigación. En Texas nos enfocamos en poner en práctica lo que aprendimos en la Casa de Tepetla y en UcsB nos centramos en entender por qué los estudiantes latinos no se graduaban, no llegaban a las universidades y no terminaban sus estudios. Al mismo tiempo que aprendíamos, interveníamos con el objetivo de superar las barreras, tratando de aplicar lo aprendido con el fin de reclutar y retener a los estudiantes latinos en las universidades y colegios comunitarios del área. En la Casa de Tepetla los estudiantes formados durante "la edad de oro" aprendimos a hacer investigación investigando. No interveníamos para resolver los problemas sociales que enfrentaban nuestros sujetos de estudio, pero sí los discutíamos con nuestros profesores y debatíamos sobre la impotencia que sentíamos al no poder solucionarlos. Nuestros docentes, distinguidos profesores-investigadores y sus asistentes, los estudiantes del posgrado en antropología de la UIA, hacíamos trabajo de campo juntos, vivíamos un tiempo en la Casa de Tepetla y luego regresábamos a vivir a nuestras comunidades. En la Casa "Ángel Palerm" de Dallas los profesores éramos antropólogos y había algunos estudiantes de antropología, pero muchos otros colegas y estudiantes provenían de disciplinas como sociología, ciencias políticas, justicia criminal, educación, geografía y psicología. Pocos vivíamos en la Casa “Ángel
Palerm" y la gran mayoría vivía con sus familias en el mismo barrio o en los barrios vecinos y nos visitaban al menos dos veces por semana.

En la Universidad de Guanajuato también aplicamos lo aprendido en la Casa de Tepetla para trazar y medir el tamaño y composición de las redes que construyen los migrantes guanajuatenses en Estados Unidos. La esencia del modelo utilizado fue y sigue siendo la misma: la investigación in situ, de larga duración, basada en el uso de una estación de campo permanente instalada en la comunidad bajo estudio, en la cual se capacitan estudiantes al mismo tiempo que se recaba, registra y analiza información de forma científica, y donde los participantes establecen relaciones de confianza y discuten los problemas de la población, la comunidad y la región. Tanto la Casa de Tepetla como la Casa "Ángel Palerm" albergaron los proyectos de investigación de sus profesores. En los dos lugares enseñamos y aprendimos los métodos tradicionales de la antropología social y la etnografía, y en ambos generamos valiosa información sobre asuntos clave.

\section{BIBLIOGRAFÍA}

Alexander, Bobby C., Víctor García, Laura González, Geoffrey Grimes y Dan O’Brien, 2003, “New Challenges for Community Colleges: Latino Immigrants and the Transfer Process", Conference on Community Colleges and Latino Educational Opportunity, octubre, Boston.

, 2007, "Barriers in the Transfer Process for Hispanic and Hispanic Immigrant Students", en Journal of Hispanic Higher Education, vol. 6, núm. 2, pp. 174-184.

Alonso, Jorge, 1999, Ángel Palerm Vich. Innovador de la antropología mexicana, Colegio de Etnólogos y Antropólogos Sociales (Serie Bibliografías, núm. 1), México.

Cátedra "Ángel Palerm”, 2012, “Cátedra Ángel Palerm 2012", disco compacto, Universidad Iberoamericana, Centro de Investigaciones y Estudios Superiores en Antropología Social, México.

García, Víctor, 2001, "Bringing Anthropology Home: Latino Students, Ethnographic Research, and Us Rural Communities", Julian Samora Research Institute-Michigan State University (Occasional Paper, núm. 57), East Lansing. 
2002, "Mexican Enclaves in Southern Chester County, Pennsylvania: Revisiting Old Observations of an Ongoing Immigration Process", en Gilbert García y Jerry García (eds.), Readings in Chicano Studies, Kendall, Hunt Publishing Company, Iowa.

2003, "Substance Abuse Research among Transnational Mexican Farmworkers: Obstacles and Suggestions for Overcoming Them", en Juana Mora y David Díaz (eds.), Research in Action: A Participatory Model for Advancing Latino Social Policy, Haworth Press, Nueva York.

- y Laura González, 2009, "Juramentos and Mandas: Traditional Catholic Practices and Substance Abuse in Mexican Communities of Southeastern Pennsylvania", en NAPA Bulletin, vol. 31: Immigration.

__ 2011, "Participatory Research Challenges in Drug Abuse Studies among Transnational Mexican Migrants", en Journal of Open Anthropology, vol. 4: Farmwork and Social Adversity.

-y Geofrrey Grimes, 2004a, "How Cultural Studies Help Latino Students Transfer to Universities", en The Community College Enterprise, vol. 10, núm. 2, pp. 1-17.

— 2004b, "Using Ethnography in the Community College as a Strategy to Recruit Latino Students to the University", en Teaching Anthropology: Society for Anthropology in Community Colleges Notes, vol. 11, núm. 1, pp. 61-77.

García Acosta, Virginia (coord.), 2000, La diversidad intelectual. Ángel Palerm in memoriam, Centro de Investigaciones y Estudios Superiores en Antropología Social, México.

Glantz, Susana, 1987a, “Ángel Palerm (1917-1980)”, en Susana Glantz (comp.), La heterodoxia recuperada. En torno a Ángel Palerm, Fondo de Cultura Económica, México, pp. 13-47.

- (comp.), 1987b, La heterodoxia recuperada. En torno a Ángel Palerm, Fondo de Cultura Económica, México.

González, Laura, 2003a, "The Golden Years of Mexican Anthropology at the Iberoamericana University", inédito ["Los años dorados de la antropología mexicana en la Universidad Iberoamericana”].

__ 2003b, "La mejor manera de formar investigadores es investigando", en Inventario Antropológico, Anuario de la Revista Alteridades, vols. 8 y 9, Universidad Autónoma Metropolitana-Iztapalapa.

_ 2003c, "The Best Way to Train Researchers is by Doing Research. La Meilleure Façon de Former un Chercheur est en Faisant de la Recherche", material distribuido en la inauguración del Aula "Ángel Palerm", Universidad Iberoamericana, mecanoescrito.

_ 2006, "A Strategy to Overcome Obstacles Latinos Face in Higher Education”, en TACHE Noticiario, vol. 17.
Iris, Madelyn, 2004, "Introduction: What is a Cultural Anthropology Field School and What is it Good For?", en NAPA Bulletin, vol. 22, núm. 1, pp. 8-13.

Mid-Atlantic Addiction Research and Training Institute (MARTI), "Research Projects and Publications by Dr. Victor Garcia”, en línea: <http://www.iup.edu/page.asp $\mathrm{x}$ ?id=52211 $>$.

Palerm, Jacinta, 1997, "El trabajo de campo y la formación de investigadores en antropología social", en Nueva Antropología. Revista de Ciencias Sociales, núm. 51, febrero.

Palerm, Juan Vicente, 1999, "Las nuevas comunidades mexicanas en espacios rurales de los Estados Unidos de América: a propósito de una reflexión acerca del quehacer antropológico", en Áreas. Revista de Ciencias Sociales, núm. 19.

Rojas Rabiela, Teresa, 1987, "La familia dividida y los círculos invisibles: las instituciones y los estudios de posgrado en antropología en México", ponencia, México.

Suárez, Modesto (coord.), 1990, Historia, antropología y política. Homenaje a Ángel Palerm, 2 vols., Universidad Iberoamericana, Alianza Editorial, México.

Torres, Patricia, 1988, “Ángel Palerm Vich”, en Lina Odena Güemes y Carlos García Mora (coords.), La antropología en México, panorama histórico, vol. II: Los protagonistas (Nájera-Yurchenco), Instituto Nacional de Antropología e Historia, México, pp. 117-143.

United States Census Bureau, 2002, “QT-PL, Race, Hispanic or Latino, and Age: 2000”, en línea: <http://factfinder. census.gov $>$.

_ 2003a, "2000 Census of Population and Housing Summary File 1 Characteristics for Zip Codes (75203, 75208, 75211, 75212, 75216, 75224, 75232, 75233, 75236, 75237, 75241, 75249)", en línea: <http://census. dfwinfo.com/sf3/dp2.asp?Geo=Zipcode\&Code $=>$. , 2003b, "Census 2000 Profile of Selected Economic Characteristics for Zip Codes (75203, 75208, 75211, 75212, 75216, 75224, 75232, 75233, 75236, 75237, 75241, 75249)", en línea: <http://census.dfwinfo.com/sf3/dp2. asp?Geo=Zipcode $\&$ Code $=>$.

, 2003c, "Census 2000 Profile of Selected Social Characteristics for Zip Codes (75203, 75208, 75211, 75212, 75216, 75224, 75232, 75233, 75236, 75237, 75241, 75249)", en línea: <http://census.dfwinfo.com/ sf3/dp2.asp?Geo=Zipcode\&Code $>$.

Vázquez León, Luis, 1996, "Ángel Palerm y la institucionalización de la antropología social en México”, en Alteridades, núm. 15.

Viqueira, Carmen, 1999, "Algunas experiencias sobre la enseñanza de la antropología social en México”, en Áreas. Revista de Ciencias Sociales, núm. 19.

Wolf, Eric, 1981, “Obituaries: Ángel Palerm Vich, 1917-1980”, en American Anthropologist, núm. 83, pp. 612-615. 